\title{
Particles of man (part I)
}

\author{
Giulio Maltese
}

Published online: 26 September 2013

(c) Centro P.RI.ST.EM, Università Commerciale Luigi Bocconi 2013

\begin{abstract}
This article - the first of a three-part seriesaims to briefly retrace the milestones in the development of particle physics, which led to what we believe is a consolidated framework, manufactured by man to explain what he himself observes, in his role as part of nature.

Keywords History of particle physics · Quantum mechanics · P. A. M. Dirac · Quantum electrodynamics · Enrico Fermi · Beta decay $\cdot$ Strong interactions · Weak interactions · Isotopic spin · Pion · Muon · Strange particles · Hadrons · Eugene Wigner
\end{abstract}

\section{The beginnings}

In 1930 physics was very different from how it looks today. Only two forces (gravity and electromagnetism), and three particles (the proton, the electron and the photon) were known at that time. The preceding few decades had witnessed the thunderous birth of novel theories and a series of striking discoveries: from X-rays (Wilhelm C. Röntgen, 1895) to radioactivity (Henri Becquerel, 1896), from the electron (Joseph J. Thomson, 1897) to the quantum hypothesis (Max Planck, 1900), Albert Einstein's theories of special (1905) and general relativity (1915), and the conjectural existence of the quantum of light, i.e., the photon (Einstein, 1905), confirmed experimentally by Arthur H. Compton in $1923 .{ }^{1}$ In the years following its formulation the quantum hypothesis would go on to be applied to atomic systems of growing complexity-and with increasing difficulties-using semi-classical criteria.

G. Maltese $(\bowtie)$

Via Tiberio Imperatore 65, 00145 Rome, Italy

e-mail: giulio_maltese@libero.it
This was the scenario until the emergence of quantum mechanics (1925-1927), mainly due to Werner Heisenberg, Max Born, Pascual Jordan (Germany), Erwin Schrödinger (Austria) and Paul A. M. Dirac (Britain), unveiled a revolutionary way of thinking, and allowed for the atomic and molecular phenomena known at that time to be organised in a harmonic and coherent framework (Fig. 1). ${ }^{2}$

In 1927 Dirac [1] created the first quantum field theory by applying the methods of quantum mechanics to the electromagnetic field, which he described as a collection of photons (Fig. 2). When interacting with the field an atom can either absorb a photon, thus reaching an excited level, or emit it, and pass from an excited state to a state of lesser energy. The photon, or quantum of light, is the channel through which the electromagnetic field interacts with atoms of matter, and the idea that a quantum field can be described by a particle 'carrying' or 'mediating' the interaction would prove lasting and fruitful. Another ground-breaking consequence was that the electromagnetic field could be described by a variable number of particles (photons) that could 'appear' and 'disappear', in other words be 'created' and 'annihilated' as a result of the processes of absorption and emission.

\footnotetext{
${ }^{1}$ Röntgen, Becquerel and Thomson were awarded the Nobel Prize in 1901, 1903 and 1906, respectively; Planck, Einstein and Compton in 1918, 1921 and 1927. To this list we add Niels Bohr, who won in 1922 for the first model of the atom (1913) based on quantum hypotheses.

2 The work of David Hilbert and Johann Von Neumann was decisive for a systematic understanding of the foundations of quantum mechanics: it appeared in a famous series of lectures on the development of quantum theory held in Göttingen in the winter 1926-1927. Heisenberg received the Nobel Prize in 1932; Schrödinger and Dirac shared the 1933 Prize. Born was awarded only in 1954.
} 
With quantum electrodynamics (QED) the concept of field, born in the nineteenth century thanks to Maxwell's electromagnetism, and which was later to be taken by Einstein as the foundation for general relativity, entered the quantum formalism. ${ }^{3}$

In 1928 Dirac presented the equation that now bears his name; using this he obtained a quantum theory for the electron in agreement with the principles of special relativity, and that made it possible to view the electron's $\operatorname{spin}^{4}$ as a natural by-product of the theory. The remarkable success of the latter had, as a counterpart, the appearance of states of negative energy, which seemed to have no physical meaning. Dirac conjectured that negative-energy states were the most stable ones for electrons, and that they were all filled. The exclusion principle says that any state may be occupied by one electron at most, so that electrons will fill increasingly unstable states. In vacuum, viewed as a sea of states with negative energy, there might be gaps, manifesting themselves as positively charged particles. Dirac initially proposed to identify these with protons; finally, in 1931, in the face of strong resistance to his hypothesis, he suggested treating the 'gaps' as particles with the same mass as electrons and positive charge, which he called antielectrons. When an electron filled a gap, the electron and the antielectron annihilated each other, thus emitting energy. Predicting the existence of the first antimatter particle made Dirac's theory fascinating but extremely daring.

\footnotetext{
${ }^{3}$ Pascual Jordan had made important preliminary contributions in this direction. Enrico Fermi, with the typical clarity he was known for, explained the subtleties of the new theory in an article published in 1932 [2] from which generations of young scientists learned quantum electrodynamics.

4 The term spin was initially introduced to denote the intrinsic angular momentum of the electron, conjectured to exist (1925) by the Dutch physicists Samuel A. Goudsmit and George E. Uhlenbeck. The spin $s$ is measured in $\hbar$ units ( $\hbar=h / 2 \pi ; h$ is Planck's constant). There are two kinds of particles: bosons, with integer spin $(s=0,1,2, \ldots)$, thus called because they obey the Bose-Einstein statistics, and fermions, with semi-integer spin $(s=1 / 2,3 / 2,5 / 2, \ldots)$ and characterised by the Fermi-Dirac statistics. The electron is a fermion with spin $1 / 2$. The spin component along a given direction, for example the $z$-axis, is denoted by $s_{\mathrm{z}}$ and can equal $\pm 1 / 2$ for the electron. The double-valued spin quantum number was introduced by the Austrian Wolfgang Pauli to account for spectroscopic data. Fermions follow the exclusion principle, formulated by Pauli in 1925: in a system of interacting identical fermions (for example the electrons in an atom) no two can have the same quantum numbers simultaneously. Bosons do not obey Pauli's principle. Quantum statistics were elaborated between 1924 and 1926: they consider particles as indistinguishable. In classical physics (where particles can be distinguished) the Maxwell-Boltzmann statistics holds, and either quantum statistics reduce to it at high temperatures or low densities, where the waves associated to the particles don't interfere with each other and the quantum effects are absent. For formulating the principle that bears his name Pauli received the Nobel Prize in 1945.
}

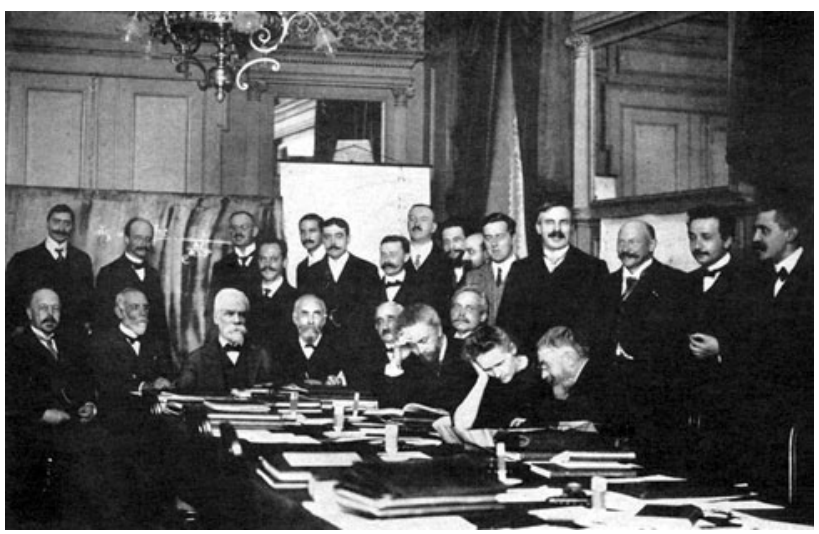

Fig. 1 Participants in the fifth Solvay Conference, held in Brussels in October 1927. These meetings, sponsored by the Belgian industrialist Ernest Solvay, were important opportunities for discussions and progress. Standing (from left): A. Piccard, E. Henriot, P. Ehrenfest, E. Herzen, Th. de Donder, E. Schrödinger, J. E. Verschaffelt, W. Pauli, W. Heisenberg, R. H. Fowler, L. Brillouin. Seated (back row): P. Debye, M. Knudsen, W. L. Bragg, H. A. Kramers, P. A. M. Dirac, A. H. Compton, L. de Broglie, M. Born, N. Bohr. In the front row: I. Langmuir, M. Planck, M. Skłodowska-Curie, H. A. Lorentz, A. Einstein, P. Langevin, Ch.-E. Guye, C. T. R. Wilson, O. W. Richardson. The 1927 conference, entitled 'Electrons and photons', was by and large devoted to quantum mechanics, which had already conquered the main stage. Of the 29 participants, 17 were or would become Nobel Prize winners. Photo: Benjamin Couprie, 1911

\section{The Quantum Theory of the Emission and Absosption of Radiation. \\ By P. A. M. Drrac, St. John's College, Cambridge, and Institute for Theoretical Physics, Copenhagen. \\ (Communicated by N. Bohr, For. Mem. R.S.-Received Fobruary 2, 1927.)}

\section{§1. Introduction and Summary.}

The now quantum theory, based on the assumption that the dynamical variables do not obey the commutative law of multiplication, has by now been developed sufficiently to form a fairly complete theory of dynamias. One can trest mathematically the problem of any dynamical system composed of a number of particles with instantaneous forces acting between them, provided it is describs ble by a Hamiltonian function, and one can interpret the mathematicy physically by a quite definite genersl method. On the other band, hardly anything has been done up to the present on quantum electrodynamics. The questions of the correct trestment of a system in which the forces are propagated with the velocity of light instead of instantaneously, of the production of an electromagnetic field by a moving electron, and of the reaction of this field on the electron have not yet been touched. In addition, there is a serious difficulty in making the theory satisfy all the requirements of the restricted

Fig. 2 Part of the first page of Dirac's 1927 paper [1] on quantum electrodynamics, presented to the Royal Society by Niels Bohr

\section{Towards the atomic nucleus}

With the success of quantum mechanics it became clear that the frontier of physics was edging towards the nucleus of the atom: but here darkness prevailed. Known were the radioactive elements discovered by Pierre and 
Marie Curie (who shared the 1903 Nobel Prize in physics; Marie Curie also won the Chemistry Prize in 1911) and the various types of radioactivity (alpha, beta, gamma): actually, the study of alpha radioactivity had been decisive for the introduction of Rutherford's nuclear model of the atom in 1911, which superseded the earlier version of Thompson where the atom was represented as a positively-charged ball containing the electrons.

We owe to Ernest Rutherford (born in New Zealand, 1908 Nobel Prize in chemistry) the introduction of the concept of the atomic nucleus. However, this idea carried with it controversial issues. The first of these controversies concerned its structure: it was thought that a nucleus of mass $A$ and atomic number $Z$ would be made of $A$ protons and $A-Z$ electrons. Electrons 'had' to live inside nuclei: wasn't it nuclei that emitted electrons during beta decay? Yet the presence of electrons in the nucleus spawned serious theoretical objections. There was also the mystery of the nitrogen nucleus: having an atomic weight of 14 it 'had' to obey the Fermi-Dirac statistics, for it contained an odd number of particles with semi-integer spin (14 protons and 7 electrons). But in 1929 Franco Rasetti, a physicist from Fermi's team, proved that the nucleus ${ }^{14} \mathrm{~N}$ actually followed the Bose-Einstein statistics, which meant it consisted of an even number of particles with semi-integer spin. Not much long after that, other measurements showed that the nucleus ${ }^{6} \mathrm{Li}$ also obeyed the 'wrong' statistics.

A second puzzle was that the energy of the electrons emitted during beta decay varied continuously over a given interval. This implied that either the conservation of energy wasn't valid in single nuclear processes, as Bohr had started to maintain in 1929, or during beta decay not only electrons were emitted, but other particles as well. Towards the end of 1930, in the 'desperate' attempt to overcome the problem posed by nuclear statistics and the continuous beta spectrum, Pauli conjectured that the nucleus contained particles with no charge, spin $1 / 2$ and very small mass. He suggested these particles were emitted together with electrons, thus 'rescuing' conservation laws in single elementary processes. Nowadays we are used to the jumble of subatomic particles, but back then there were very few. Postulating a new particle was brave, but as Pauli wrote, 'nur wer wagt, gewinnt' (only he who dares wins).

\section{From the neutron to the theories of beta decay and the nuclear force}

The international conference held in Rome in October 1931 was one important step for the Fermi group in the direction of nuclear physics. ${ }^{5}$ The prevailing climate of those times echoes in Guglielmo Marconi's opening speech:

"The assault lead by Man on the Atom, this tiny yet impenetrable stronghold, has already shown encouraging and surprising results. The outermost fortifications have already given in ..., but the inner core, the atom's nucleus, remains, at least in part, still a mystery." 6

Nevertheless, crucial advances followed shortly. In early 1932 the British physicist James Chadwick discovered the neutron. In August of that year, while observing cosmic rays - an ionising radiation made in part by an emission from outer space and in part generated by the interaction of the latter with the atmosphere ${ }^{7}$ - the American scientist Carl D. Anderson detected a particle with the same mass as the electron and a positive charge. The following year that particle was confirmed to be precisely the one Dirac had predicted, and was called 'positron'. Still in 1932 Heisenberg envisioned a model of the nucleus made of protons and neutrons, and suggested that these could be thought of as two states of the same entity, the nucleon.

At the end of 1933 Enrico Fermi [3] devised a quantum field theory for the beta decay, analogous to QED (Fig. 3). He defined a new type of force, the so-called weak interaction, that was responsible for decay, and whose fundamental process was transforming a neutron into a proton, an electron and a neutrino (the particle forecasted by Pauli): $n \rightarrow p+e+v_{e}$ (now we know that an antineutrino $\bar{v}_{e}$ is emitted instead of $v_{e}$ ).

Fermi accepted the idea of Heisenberg mentioned above. The novelty was in assuming that the pair electronneutrino doesn't exist in the nucleus prior to beta decay; rather, it is created precisely when decay occurs, just as we can't think that the quantum of light emitted by an atom during a quantum leap exists inside the atom before it is emitted. It is not therefore necessary to assume electrons inside the nucleus - which consists of protons and neutrons as originally thought - and the continuous spectrum of the electrons of beta rays can be explained by the emission of a

\footnotetext{
5 The first International Conference on Nuclear Physics, organised by Fermi and sponsored by the Volta Foundation of the Accademia d'Italia, took place in Rome 11-17 October 1931 and was attended by the most prominent physicists of this area.

${ }^{6}$ Atti del Convegno di Fisica Nucleare, Roma, Reale Accademia d'Italia, 1932, p. 11. The Conference had a conspicuous press coverage: see for example La Tribuna of October 13. Mussolini received the participants to the Conference upon its closure, on October 17.

7 Using aerostats the Austrian Victor Hess discovered in 1912 that the level of the ionising radiation increased with altitude, hence excluding a terrestrial origin. Later it became clear that cosmic particles had two sources: primary cosmic rays from outer space, and the secondary component produced by the interaction with the atmosphere. For the discovery of the positron Anderson shared with Hess the Nobel Prize in 1936, while Chadwick won in 1935.
} 
third particle, after proton and electron, as Pauli had predicted. During the opposite process (from proton to neutron) a pair electron-neutrino disappears, similarly to when a light quantum is absorbed as an atom jumps to a state of higher energy. ${ }^{8}$

In 1934-1935 the Japanese Hideki Yukawa (Nobel Prize in 1949) established the first theory of the 'strong' interaction, which keeps nuclei together, by relying once again on a quantum field theory. He assumed that the interaction between nucleons was due to the exchange of a particle with integer or zero spin and three possible charges: positive, negative and neutral. The neutral particle should be emitted or absorbed during proton-proton $(p p)$ and neutron-neutron $(n n)$ interactions, whereas charged particles would arise or be destroyed in proton-neutron interactions (pn). The electromagnetic interaction has infinite range and is described by the Coulomb potential $V(r)=1 / r$, where $r$ is the distance. The infinite range of the interactions is associated to the zero mass of the photon. The range of nuclear interactions, instead, is bounded (around $10^{-13} \mathrm{~cm}$ ), hence the particle carrying the interaction has finite mass. We can imagine that the interaction consists of a nonstop exchange of 'virtual' particles of mass, say, $m$ : every nucleon is surrounded by its own field, and emits and absorbs particles of mass $m$ continuously. Since creating a particle has an energy cost, equal to $\Delta E=m c^{2}$, one can somehow 'borrow' energy, in other words violate the law of conservation of energy, as long as the 'loan' lasts a very short time $\Delta t$ (equal to $\hbar / \Delta E$ ). This is possible, by the uncertainty principle, because the time and energy variables satisfy the uncertainty relation $\Delta E \Delta t \geq \hbar$ : in order to measure the quantity of energy $\Delta E$, we need at

\footnotetext{
${ }^{8}$ The year 1934 witnessed the Fermi group's big experimental successes. After artificial radioactivity, produced by alpha particles and discovered by the French couple Frédéric Joliot and Irène Curie (daughter of Marie, and 1935 Nobel Prize winner in chemistry with her husband), Fermi and his team decided to try with neutrons, given their better ability to penetrate the nucleus by lack of charge. In March 1934 Fermi discovered neutron-induced artificial radioactivity, and in October he found that the excitation power of neutrons grew enormously if these were slowed by forcing them to go through a hydrogenated substance, like water or paraffin. Slow neutrons spent more time inside the nucleus, increasing the probability of nuclear interaction, and hence of activation as well. For these discoveries Fermi was awarded the Nobel Prize in 1938. Immediately after he moved to the US because of the Racial Laws (his wife Laura was Jewish) and because of the poor prospects research had in Fascist Italy. He lectured at Columbia University (New York) and then Chicago. He was the first to achieve the nuclear chain reaction in 1942, and was a central figure of the Manhattan Project, on which thousands of scientists worked to build the atomic bomb. In the postwar period he returned to Chicago to study 'fundamental' particles (he disliked the name 'elementary', a term he found increasingly inadequate as new particles were constantly discovered). After the Rome group he created a second School of physics in Chicago, where many brilliant students and future Nobel Prize winners were formed. Several of them will be mentioned in this article.
}

\section{LA RICERCA SCIENTIFICA} ED IL PROCRESSO TECNICO NEL': ECONOMUA NUDONALE

\author{
Tentativo di una teoria dell' emissione \\ dei raggi "beta" \\ Noce del poot. ENRICO FERMI
}

Rinsunto: Teoria della emissione dei raggi is delie sostanze radioattive, foodata sulPipotesi che gli elettroni emessi dai nuclei noa esistano prima della disintegrazione ma vengano formati, insieme ad un neutrinc, in modo analogo alla formazione of teoria con l'esperienza.

Mi propongo di esporse qui i fondamenti di una teoria dell'emissione dei raggi $\beta$ che. lenche basata sopra ipotesi delle quali nanca al momento presente qualsiasi conferma sperimentale. sembra tuttavia capace di dare una rappresentazione abbastanza accurata dei fatti e permette una tratta. zione quantitativa del comjoraniento degli elettroni nucleari che. se pure le ipotesi fondamentali della teoria dovessero risultare false, potrà in onni caso servire di utile guida per indirizzare le ricerche sperimentali.

E' ben noto che nel cercare di costruire una teoria dei raggi $\beta$ si incontra una prima difficolta dipendente dal fatto che $i$ raggi $\beta$ escono dai nuclei radioattivi con una distribuzione continua di velocita che si estende nino a una certa velocità massima : ciò che a prima vista non sembra conci. liabile col principio della conservazione dell'energia. L'na possibilitè qualita. tiva di spiegare i fatti senza dovere abbandonare il principio della conser. vazione dellenergia consiste, secondo Pauli, neil ammettere liesistenza del cosi detto "neutrino n, e cioé di un corpuscolo elettricamente neutro con massa dellordine di grandezza di quella dell'elettrone o minore. In ogni disintegrazione $\beta$ si avrebbe emissione simultanea di un eletrone e di un neutrino: e lenergia liberata nel processo si ripartirebbe comunque tra due corpuscoli in modo appunto che lenergia dell eiettrone possa prendere tutti $i$ valori da 0 fino ad un certo massimo Il neutrino d'altra parte. a causa della sua neutralitá elettrica e della piccolissima massa avrebbe un potere penetrante cosi elevato da suggire praticamente ad ogni attuale metc penetrante cosi elevato da sfuggire praticamente ad ogni attuale metodo di osservazione. Nella teoria che ci proponiamo di esporre ci metteremo dal punto di vista della ipotesi dell'esistenza del neutrino.

Fig. 3 Enrico Fermi and the first page of the Italian version of his work on the theory of beta decay [3]. The article was republished in 1934 in the more prestigious journals Nuovo Cimento [4] and Zeitschrift für Physik [5]. Hideki Yukawa was the first to propose a quantum field theory for the strong nuclear force in the paper 'On the interaction of elementary particles' [10]

least $\Delta t$ time, and within $\Delta t$ we can't 'see' the missing energy $\Delta E$. Now, in time $\Delta t$ the particle carrying the nuclear interaction can go as far as $R=10^{-13} \mathrm{~cm}$ at most, exactly the range of the nuclear force observed in the experiments. Therefore we have $c \Delta t=R=\hbar / m c$, which permitted the estimation that $m$ is about 200 times the mass of the electron. In Yukawa's theory the finite range of nuclear forces prompted the consideration of the potential $V(r)=g^{2}\left(e^{-r / R}\right) / r$, replacing the Coulomb potential $V(r)=1 / r$, where $g$ is the so-called 'coupling constant' of the interaction.

In 1936 it was proved, within the range of experimental errors, that the $n n, p p$ and $n p$ forces have the same magnitude: the strong force seemed to be independent of the electrical charge. As we mentioned earlier, according to 
Heisenberg's nuclear theory both proton and neutron were states of a unique particle, the nucleon, depending on the values of an internal quantum number that in 1937 the Hungarian Eugene P. Wigner labelled isotopic spin. ${ }^{9}$ Today we call it isospin ( $I$ in symbols). We may then think of the nucleon as a particle with isospin: it admits two possible states, protonic and neutronic, corresponding to the possible values of the component of the isospin along a fixed direction in an abstract space. The notion of isospin proved to be particularly relevant, and would later be generalised to become a crucial ingredient in the classification of particles. We can visualise the isospin as a vector of components $I_{1}, I_{2}, I_{3}$ in an abstract three-dimensional space: the experimental discovery that nuclear forces don't depend on electric charge implies that they are invariant under rotations in the space of isospin vectors. In all processes involving strong interactions, therefore, the isospin is preserved: this means that the length of the $I$ vector doesn't change, and the only thing that may vary is the value of the components, e.g., $I_{3}$.

We can also see this from another point of view. Albeit different in charge, proton and neutron have almost equal mass (respectively 938.27 and $939.57 \mathrm{MeV} / c^{2}$ ). ${ }^{10}$ This fact indicates that the particles' Hamiltonian (the quantum operator, associated to the energy of a physical system, that plays an essential role in describing the system's evolution) has energy levels occurring more than once, or as we say, it has degenerate (or almost degenerate) energy levels. Now, if a quantum system has degenerate energy levels its Hamiltonian is invariant under some transformation, and this invariance heralds the existence of a conserved quantity. Thus, in particle physics each time we have a number of particles with comparable mass it may be worth seeking invariants, for the similar masses are nothing but (almost) degenerate energy levels. ${ }^{11}$ This is what happens to the proton-neutron system, which forms a 'doublet' with isospin $I=1 / 2$ and $I_{3}= \pm 1 / 2$ (plus sign for the proton and minus for the neutron). The existence of 'multiplets' of differently charged particles having almost the same mass must be related to the invariance properties under rotations in the isospin space. The transformations generating the

\footnotetext{
9 Wigner won the Nobel Prize in 1963 for his contributions to nuclear and particle physics, and in particular for the discovery and application of crucial symmetry principles.

${ }^{10}$ Particle physics, where mass and energy are often employed as synonyms (due to the relation $E=m c^{2}$ ), normally uses the Electronvolt as unit for the energy $(1 \mathrm{eV}$ is the energy absorbed by an elementary charge, such as a proton or an electron, when going through an electrical field with voltage 1 Volt), and $\mathrm{eV} / \mathrm{c}^{2}$ or its multiples $\left(\mathrm{MeV} / c^{2}, \mathrm{GeV} / \mathrm{c}^{2}\right)$ as units of mass.

11 This holds for proton and neutron up to corrections due to difference of charge. In fact, electromagnetic forces (such as the weak ones) don't preserve the isospin: the electromagnetic properties tell proton and neutron apart.
}

rotations in the isotopic spin space form a group, in this case the group SU(2) (see Sect. 7).

\section{From the mesotron to the pion}

In 1937 the Americans Carl Anderson and Seth NedderMeyer observed a particle in the cosmic radiation, which they called 'mesotron', of mass similar to the prediction of Yukawa's theory. However, in 1946 news spread about the experiments three Italian researchers-Marcello Conversi, Ettore Pancini and Oreste Piccioni-had carried out during the war, amid innumerable difficulties. Their tests showed that the 'mesotrons' detected in cosmic rays interacted with nuclei far too weakly to be the quanta carrying the strong force. According to the hypothesis of the 'double meson', put forward in 1947, the mesotron wasn't Yukawa's particle, but its decay output. During exactly during those same months, scientists detected in cosmic rays the particle predicted by Yukawa, which was given the name of ' $\pi$ meson' or pion. The 'mesotron' then became the ' $\mu$ meson', and eventually was named muon. A positively charged meson decayed to a muon according to the rule $\pi^{+} \rightarrow \mu^{+}+v$. The era of high-energy nuclear physics had begun.

The theory of the double meson didn't explain everything, quite the contrary. The muon issue was still unsolved. The story goes that upon hearing about muons the American physicist Isidor I. Rabi (Nobel Prize in 1944) said: 'Who ordered that?' That the muon's role in the universe was simply that of product of the decay of the pion seemed too restrictive. All other particles, like the electron or the proton, played a well-defined part in the structure of atoms and nuclei. But this was, after all, the warning of a more complex pattern, whose surface 1947 physics was only scratching. Murray Gell-Mann, one of the creators of contemporary theoretical physics, wrote in 1958: 'The muon was the unwelcome baby on the doorstep, signifying the end of days of innocence' [7].

\section{Advances in quantum electrodynamics. Accelerators and the first 'home-made' particles. Strangeness}

The other big topic being discussed by physicists in 1947 was the so-called Lamb shift (after Willis E. Lamb, who was awarded the Nobel Prize in 1955 for this discovery), a deviation in the hydrogen spectrum between energy levels that should actually be the same according to QED.

Ever since 1930 scientists had realised that the computations of certain physical quantities made within the framework of QED gave contrasting results, a clear indication that something wasn't right. The debate on the Lamb 
shift became the starting point for the so-called 'renormalisation programme' of QED, championed by the Americans Richard P. Feynman and Julian Schwinger, the Englishman Freeman Dyson and the Japanese Shin-Ichiro Tomonaga. Feynman, Schwinger and Tomonaga received the Nobel Prize in 1965. The renormalisation programme didn't remove infinitely large values from the theory; it confined them to controlled situations, thus warranting finite previsions to any order of accuracy, provided the mass and the charge of the electron were 'renormalised', and similarly for any quantity associated to the quantum field. Renormalising means assigning to divergent expressions certain a priori values, fixed by physical considerations; for instance, decreeing that the electron's charge and mass take the values observed in reality. The need to renormalise the electronic mass and charge implies that the theory can't predict their values, but has to read them off experimental results: put differently, they are phenomenological parameters. However, this is a small price to pay for a theory that over time has proved to be one of the most reliable, from the point of view of experimental predictions, ever produced by man.

In the meantime, the post-war period had witnessed the arrival of 'Big Science', a change of scale in physics that followed the expansion of research groups and the increase of budgets. The construction of more and more powerful accelerators led in 1948 to the first 'artificial' mesons, and two years later to the first discovery of a particle using an accelerator (the neutral pion $\pi^{0}$, governing $p p$ and $n n$ interactions). The $6.2 \mathrm{GeV}$ Bevatron in Berkeley, inaugurated in 1954, was where Emilio Segrè and Owen Chamberlain observed the antiproton; they shared the Nobel Prize in 1959 for this discovery. ${ }^{12}$

In 1947 physicists noticed certain phenomena in cosmic rays that left $V$-shaped traces in particle detectors. They started talking of $V$-particles or strange particles because of their behaviour. The main problem was how to mediate between a high occurrence rate (indicating the strong force was the cause) and a long half-life (proving that they decayed under the weak force ${ }^{13}$ ). The key to solving the riddle was found in 1953 by Murray Gell-Mann and, independently, Kazuhiko Nishijima. It relied on the conservation of isospin in strong interactions and on the introduction of a new quantum number $S$, the strangeness, that is preserved by strong and electromagnetic forces but not under weak interactions. According to the new scheme

\footnotetext{
12 A member of the Fermi group, Emilio Segrè had to move to California in 1938 after being expelled from Italian universities because of the Racial Laws.

${ }^{13}$ For example, the lambda hyperon $\Lambda^{\circ}$ was produced by the reaction $p+\pi^{-} \rightarrow \Lambda^{\circ}+\pi^{+}+\pi^{-}$(a classical strong process) with a typical reaction time of order $10^{-23} \mathrm{~s}$, and then decayed according to $\Lambda^{\circ} \rightarrow p+\pi^{-}$in a time comparable to $10^{-10} \mathrm{~s}$.
}

the proton and neutron would have strangeness 0 ; as for the other particles we met, $S=-1$ for $\Lambda^{\circ}$, and $S=1$ for $K^{\circ}$. Gell-Mann, moreover, assigned a semi-integer isospin to strange $K$ mesons, and integer isospin to strange baryons such as $\Lambda^{\circ} .{ }^{14}$ The conservation of isospin in strong interactions accounted for the observations. The decay of the hyperon $\Lambda^{\circ}$ into $p+\pi^{-}$, for example, takes a time that is characteristic of weak interactions. So why can't the strong force possibly be the cause of this decay? For $\Lambda^{\circ}$ we have $I_{3}=0$; for the proton $I_{3}$ equals $1 / 2$, while for $\pi^{-}$it is -1 . Since $1 / 2-1=-1 / 2$ is different from 0 , which is what we get on the left-hand side of the process, it follows that $\Lambda^{\circ}$ cannot decay because of strong interactions. By the same rule the reaction $n+n \rightarrow \Lambda^{\circ}+\Lambda^{\circ}$ can't occur $\left(I_{3}\right.$ is 0 for $\Lambda$ while $-1 / 2$ for the neutron). Gell-Mann and Nishijima subsequently modified the equation $Q=B / 2+I_{3}$ to incorporate strangeness: $Q=(S+B) / 2+I_{3}=Y / 2+I_{3}$. Here $Q$ is the electric charge (in $e$ units, the proton's charge), $B$ the baryon number, $Y=S+B$ the hypercharge. The picture created by strangeness, as the physicist and historian of physics Abraham Pais wrote, 'was a loyal guide during the 1950s [8].

\section{The trouble with the strong interaction}

The consolidation of quantum electrodynamics, the construction of accelerators and the discovery of Yukawa particles all instilled a certain optimism in physicists who, returning home from the war, were anxious to do pion physics with accelerators, and apply the tools that had been extraordinarily successful in QED. That confidence, however, would be short lived. The perturbative approach typical of QED was hard to apply to the strong force, given the latter's magnitude. In general we can say that the Hamiltonian in a QED problem is the sum of three terms: $H$ (electrodynamics $)=H$ (photons $)+H$ (electrons) $+e H$ (interaction). The first summand on the right describes the electromagnetic field without matter, the second accounts for electrons in absence of electromagnetic field, and the third, proportional to the elementary charge, gives the interaction between the first two. The term $e H$ (interaction) is fully known and describes the interaction of a charge moving

\footnotetext{
${ }^{14}$ Baryons (from the Greek $\beta \alpha \rho b s$, heavy) are the particles with semi-integer spin subjected to the strong force, such as proton and neutron. The baryon number $B$ is 1 for baryons, -1 for antibaryons (e.g. antiproton and antineutron) and 0 for all non-baryons, like the pion. In 1949 Wigner established the conservation principle according to which in every process the baryon number is constant. This principle has always been confirmed in the experiments so far. It could be violated, for instance, by a hypothetical protonic decay of the sort $p \rightarrow \pi^{0}+e^{+}$, which has never been observed: the experimental threshold for the half-life of the proton exceeds $5.5 \times 10^{32}$ years.
} 
must then consider either the absolute value of the $\mathrm{C}^{12}(p, p n)$ cross section or that of the $\mathrm{Al}^{27}(p, 3 p n)$ cross section (or both) to be in error. We have rather arbitrarily chosen to base our data on the $10.8-\mathrm{mb}$ value for the $\mathrm{Al}^{27}(p, 3 p n)$ cross section at $420 \mathrm{Mev}$.

Figure 1 shows that the cross section of the $\mathrm{C}^{12}(p, p n) \mathrm{C}^{11}$ reaction is a fairly insensitive function of the energy of the incident proton in the energy range studied here. Since similar results were found for the production of $\mathrm{Na}^{24}, \mathrm{Na}^{22}$, and $\mathrm{F}^{18}$ from aluminum and for $\mathrm{Be}^{7}$ formation from carbon, ${ }^{6}$ it appears to be generally true that the probability of ejecting a small number of nucleons from a small nucleus remains substantially constant over a range of bombarding energies from a few hundred Mev to at least $3 \mathrm{Bev}$. This implies that the probability that the incident particle leaves behind a relatively small amount of energy $(\approx 100 \mathrm{Mev})$ in the initial interaction with the nucleus is relatively constant over the wide energy range studied. However within this energy range meson production increases very markedly with energy and becomes a probable process. If the nucleus is large these mesons would have a good chance of being reabsorbed in the nucleus in which they were produced. This would result in a shift of the maximum in the total energy deposition spectrum to higher values, and reactions in which only a small

${ }^{B}$ Hudis, Wolfgang, and Friedlander (unpublished).

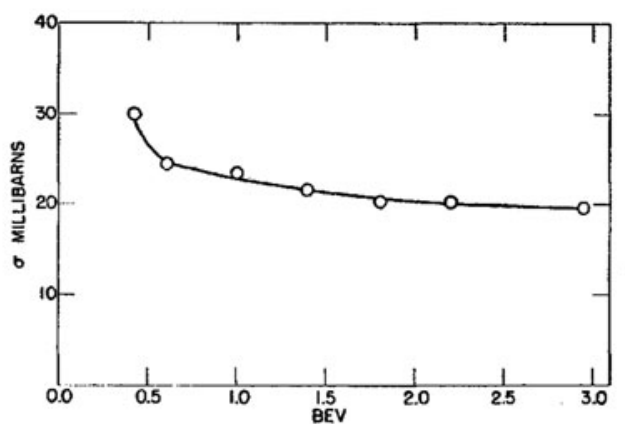

Frg. 1. Excitation function of the $\mathrm{C}^{12}(p, p n) \mathrm{C}^{11}$ reaction.

number of particles are ejected would become less likely. Such an effect has been observed in our studies on heavier nuclei. ${ }^{3}$ However, in a small nucleus reabsorption of mesons would be a much less important mode of depositing excitation energy because of their greater escape probability. Thus it becomes plausible that while the increasing dominance of meson processes decreases the cross sections for relatively simple reactions in heavy target nuclei, the cross sections for similar reactions of light nuclei remain almost unchanged.

The help of the Cosmotron operating staff is gratefully acknowledged.

\title{
Conservation of Isotopic Spin and Isotopic Gauge Invariance*
}

\author{
C. N. Yang † AND R. L. Mills \\ Brookhaven National Laboratory, Upton, New York
}

(Received June 28, 1954)

\begin{abstract}
It is pointed out that the usual principle of invariance under isotopic spin rotation is not consistant with the concept of localized fields. The possibility is explored of having invariance under local isotopic spin rotations. This leads to formulating a principle of isotopic gauge invariance and the existence of $\mathbf{a} \mathbf{b}$ field which has the same relation to the isotopic spin that the electromagnetic field has to the electric charge. The b field satisfies nonlinear differential equations. The quanta of the b field are particles with spin unity, isotopic spin unity, and electric charge $\pm e$ or zero.
\end{abstract}

\section{INTRODUCTION}

$T$ HE conservation of isotopic spin is a much discussed concept in recent years. Historically an isotopic spin parameter was first introduced by Heisenberg $^{1}$ in 1932 to describe the two charge states (namely neutron and proton) of a nucleon. The idea that the neutron and proton correspond to two states of the same particle was suggested at that time by the fact that their masses are nearly equal, and that the light

${ }^{*}$ Work performed under the auspices of the U. S. Atomic Energy Commission.

t On leave of absence from the Institute for Advanced Study, Princeton, New Jersey.

${ }^{1}$ W. Heisenberg, Z. Physik 77, 1 (1932). stable even nuclei contain equal numbers of them. Then in 1937 Breit, Condon, and Present pointed out the approximate equality of $p-p$ and $n-p$ interactions in the ${ }^{2} S$ state. $^{2}$ It seemed natural to assume that this equality holds also in the other states available to both the $n-p$ and $p-p$ systems. Under such an assumption one arrives at the concept of a total isotopic spin ${ }^{8}$ which is conserved in nucleon-nucleon interactions. Experi-

${ }^{2}$ Breit, Condon, and Present, Phys. Rev. 50, 825 (1936). J. Schwinger pointed out that the small difference may be attributed to magnetic interactions [Phys. Rev. 78, 135 (1950)].

${ }^{3}$ The total isotopic spin $\mathrm{T}$ was first introduced by $\mathrm{E}$. Wigner, Phys. Rev. 51, 106 (1937); B. Cassen and E. U. Condon, Phys. Rev. 50, 846 (1936).

Fig. 4 The first page of the paper by Yang and Mills [9] containing the first non-Abelian gauge theory. Many years later this theory would prove to be the correct gauge theory on which to build the Standard Model 
in an electromagnetic field. It is a very complicated term: what one does, then, to compute the quantities concerned, is treat the interaction term of two fields as a perturbation superposing the unperturbed fields $H$ (photons) and $H$ (electrons). In practice this entails expanding each quantity in power series in the coupling constant, called the fine-structure constant $\alpha_{E M}=e^{2} / 4 \pi \varepsilon_{0} \hbar c \approx 1 / 137$. The fact that this number is small justifies the procedure, and makes the theory's predictions highly accurate.

Yukawa's theory of the nuclear force was initially defined in analogy to electrodynamics, for it used Hamiltonians of the type $H$ (Yukawa) $=H$ (pions) $+H$ (nucleons) $+g H$ (interaction pions-nucleons). The drawback was that the parameter $g^{2} / \hbar c$ of the series expansion turned out to be very large. Furthermore, different problems seemed to give very disparate values of the parameter, killing the hope of using the perturbative approach. The beginning of the 1950s was a time of great uncertainty concerning the dynamics of strong interactions. The point was that Yukawa's mesons weren't fundamental objects, in contrast to the photons of the electromagnetic field.

In 1949 Fermi [6], with his student Chen Ning Yang, proposed the so-called 'Fermi-Yang model of the composite pion', in which they supposed the pion might not be an elementary particle but the entangled state of a nucleon with an antinucleon. The paper starts:

"In recent years several new particles have been discovered which are currently assumed to be 'elementary', that is, essentially, structureless. The probability that all such particles should be really elementary becomes less and less as their number increases" [6].

Even if the model managed to explain some features of pions, the authors realised that the hypothesis was quite reckless and they treated it as a general working reference rather than a theory capable - at least in that form-of explaining reality.

Today we know that neither pions nor nucleons are elementary: nucleons are made of three quarks and pions by a quark and an antiquark. The work of Fermi and Yang left lasting traces, though. Among the many who picked up where they left off, the Japanese Shoichi Sakata tried to generalise the idea of Fermi and Yang in 1956, by including strangeness. Sakata chose the lambda hyperon as third component in a triple including proton and neutron: together with their antiparticles, these should be the fundamental constituents of hadrons (from the Greek $\alpha \delta \rho$ ś, strong, stout), a word indicating particles subject to strong forces. Sakata's colleagues developed this idea, by noticing how the symmetry group SU(3) was the correct generalisation of the isospin group SU(2) at the heart of the FermiYang model.

\section{Symmetries, conservation laws, gauge theories}

The notion of symmetry is pivotal in modern particle physics. A physical system possesses a symmetry if a transformation doesn't alter its properties. If we consider a cylindrical vase, we see that its shape doesn't change when we turn it around. In that case we say that the vase is invariant under rotations about its axis of symmetry. On the other hand, if the vase is dented on the rim, the symmetry is broken: the effects of a rotation can be detected easily, and the rotational invariance is lost. In essence, a symmetry is the invariance of a physical system under a transformation, called symmetry transformation.

Several kinds of symmetry exist. If the vase is a cylinder, it is invariant under rotations by any angle around the axis: this is a continuous symmetry. If, instead, the shape is hexagonal, we have invariance only for rotations by $60^{\circ}$ angles or multiples: the symmetry is discrete. Next we may imagine of replicating the vase infinitely many times and place each one at a given point in space. When we rotate them simultaneously by the same angle we are performing a global transformation. By rotating each vase differently we have a local transformation.

Credit goes to Emmy Noether for realizing the importance of this property in physics. Her 1918 theorem asserts that for each physical law admitting a continuous symmetry there exists a conserved quantity. The converse statement is almost always true: every time we find a preserved quantity, there must be a corresponding continuous symmetry. In this light, the three major conservation laws in classical physics (linear momentum, angular momentum and energy) are related to certain continuous symmetries in nature: respectively, the invariance under space translations, space rotations and time translations. These symmetries correspond, in turn, to the following properties of space and time: spatial homogeneity, spatial isotropy and time homogeneity.

The symmetries of the Hamiltonian, i.e., its invariance under a group of transformations, are very important in quantum mechanics. To any given transformation corresponds a unitary operator $\operatorname{Tr}$ that acts on the wavefunction: $\psi \rightarrow \psi^{\prime}=\operatorname{Tr} \psi$. The operators of a certain type of transformations form a group. Of particular relevance are continuous transformations, those whose elements depend on a collection of continuous parameters. The unitary operators of concern will have the form $\operatorname{Tr}=\operatorname{Tr}\left(a_{1}, \ldots, a_{n}\right)$ seen as a differentiable map in $n$ variables $a_{1}, \ldots, a_{n}$. In this case one speaks of a Lie group (after the Norwegian mathematician Marius Sophus Lie, who in the second half of the nineteenth century laid out the foundations of the theory of continuous transformation groups). The origin of the parameter space conventionally corresponds to the identity 
transformation, thus $\operatorname{Tr}(0, \ldots, 0)=\mathbf{1}$. Let us assume the parameters are infinitesimal, and denote them by $\varepsilon_{i}$. By expanding up to order one we obtain the relation $\operatorname{Tr}\left(\varepsilon_{1}, \ldots\right.$, $\left.\varepsilon_{n}\right)=1-\sum_{i=1 \ldots, n} \varepsilon_{n} G_{i}$, which defines the generators $G_{i}$ of the group, Hermitian operators typically corresponding to physical observables. ${ }^{15}$

Particle physics put a great emphasis on special symmetries related to discrete transformations. One example is parity, the operation that swaps the sign of spatial coordinates and generates a new physical system that is the mirror image of the original one. The parity operator $P$, applied to the wavefunction $\psi(\mathbf{r})$, gives $P \psi(\mathbf{r})=\psi(-\mathbf{r})$. By letting it act again we return to the initial state: $P$ has eigenvalues \pm 1 and the physical system has positive $(+1)$ or negative $(-1)$ parity (provided parity is defined). The operation called charge conjugation $(C)$ transforms each particle into its antiparticle, by reversing the charge of charged particles. Also $C$ has eigenvalues \pm 1 . Lastly, the time reversal operator $T$ flips the sign of the time coordinate t: $T \psi(\mathbf{r}, t)=\psi(\mathbf{r},-t)$. If in a process due to a specific interaction $P$ (or $T$ ) is conserved, the Hamiltonian of the interaction must not involve terms that change under parity (or time reversal). Particles with spin $\boldsymbol{\sigma}$, for example, can have magnetic dipole moment $\boldsymbol{\sigma} \cdot \mathbf{B}$ since $\boldsymbol{\sigma} \cdot \mathbf{B}$ is both $P$ - and $T$-invariant. Not so for the static electric dipole moment $\boldsymbol{\sigma} \cdot \mathbf{E}$, as the latter isn't invariant under $T$. We will see in the following sections how interactions behave with respect to the operators $P, C, T$ and their combinations.

The birth of quantum mechanics made symmetries all the more significant and fascinating in the attempt to understand the natural world: it became clear that symmetry is capable of 'creating' interactions and at the same time the particles carrying them. This historical shift is realised by means of the notion of 'gauge theory', another pillar of contemporary particle physics: as a matter of fact particle physics itself consists entirely of gauge theories, as we will see.

The term 'gauge theory' refers to field theories where some transformations that do not alter the Lagrangian (the functional characterising the system, given by the kinetic energy minus the potential energy coming from the interactions) can also occur locally, and not just globally. In

\footnotetext{
15 In the present article we will come across two families of groups. The special unitary groups $\mathrm{SU}(n)$ (in particular $\mathrm{SU}(2)$ and $\mathrm{SU}(3)$, regarding the weak and strong forces) consist of $n \times n$ complexvalued unitary matrices with determinant +1 . They have $n^{2}-1$ generators and the rank of the corresponding Lie algebra (i.e. the maximal number of commuting generators) equals $n-1$. We will also mention the unitary groups $\mathrm{U}(n)$-note that $\mathrm{SU}(n)$ is contained in $\mathrm{U}(n)$ - and in particular the group $\mathrm{U}(1)$, made by $1 \times 1$ unitary matrices, when discussing the electromagnetic force. The group $U(1)$ presides over the symmetry of the electromagnetic field, in the sense that Maxwell's equations are invariant by the action of any element of $\mathrm{U}(1)$.
}

classical electromagnetism it is well known that the fields $\mathbf{E}$ and $\mathbf{B}$ are related to the vector potential $\mathbf{A}$ and the scalar potential $\varphi$ by $\mathbf{B}=\operatorname{rot} \mathbf{A}$ and $\mathbf{E}=-(1 / \mathrm{c}) \partial \mathbf{A} / \partial t-\operatorname{grad} \varphi$. The potentials $\mathbf{A}$ and $\varphi$ are not uniquely defined, since another choice like $\mathbf{A}^{\prime}=\mathbf{A}+\operatorname{grad} \chi, \varphi^{\prime}=\varphi-\partial \chi / \partial t$ (where $\chi=\chi(\mathbf{r}, t)$ ) will give rise to the same $\mathbf{E}$ and $\mathbf{B}$. This gauge invariance, expressed by potentials, had been known in physics for a long time, but it acquired a special meaning starting in the 1960s. In quantum physics the state of a system is described by the wavefunction $\psi$. The values of measurable physical quantities (the 'observables') depend on the square of the modulus of $\psi$ : therefore the theory is invariant under a map $\psi^{\prime}=\mathrm{e}^{-\mathrm{i} \alpha} \psi$ with $\alpha$ constant. This map is called a global gauge transformation because $\psi$ changes in the same way everywhere. Consequently we may choose an arbitrary phase for $\psi$.

We could also ask whether it is possible to choose the phase at each point in spacetime without affecting the theory: in fact, it is reasonable to be able to fix the conventions at one point on Earth without having to know what they are, say, on the Moon. In this case the theory should be invariant under the local gauge transformation $\psi^{\prime}(\mathbf{r}, t)=\mathrm{e}^{-\mathrm{i} \chi(\mathbf{r}, t)} \psi(\mathbf{r}, t)$. It can be shown that for this to happen, for a charged particle in an electromagnetic field, one needs to consider the transformations $\mathbf{A}^{\prime}=\mathbf{A}+(1 /$ $e) \operatorname{grad} \chi$ and $\varphi^{\prime}=\varphi-(1 / e) \partial \chi / \partial t$. The local invariance of the phase of the theory forces the existence of a field $(\varphi$, A): it is the symmetry that determines the type of interaction. The quantum carrying that field is nothing but the photon. It follows that the local phase-invariance for charged particles requires a carrier for the interaction plus an electromagnetic field of the type observed. The set of all transformations acting on the phase of the wavefunction $\psi^{\prime}(\mathbf{r}, t)=\mathrm{e}^{-i \chi(\mathbf{r}, t)} \psi(\mathbf{r}, t)$ is the unitary group $\mathrm{U}(1)_{e m}$. From $\mathrm{U}(1)_{e m}$-invariance we may infer the existence of the gauge field, identifiable with the electromagnetic field. To sum up, one can derive the equations of electrodynamics from the invariance of the equations of motion under the gauge group $\mathrm{U}(1)_{e m}$.

In general: if a particle has a certain (not necessarily electric) charge and the theory is invariant under given phase transformations, usually called gauge transformations, there must exist associated fields (gauge fields) and particles (gauge bosons). What isn't known a priori is under which gauge group the theory is invariant.

We remark that the conservation of the electric charge also has to do with gauge invariance. This aspect, first noticed by Wigner in 1949, can be explained as follows. Suppose we can create a charge $Q$ at a point in space with potential $V$ (violating charge conservation), and then move it to a point with potential $V^{\prime}<V$, thus creating the quantity of energy $Q\left(V-V^{\prime}\right)$. Now let us annihilate $Q$ (this is possible since by gauge invariance the process of regaining 
$Q\left(V-V^{\prime}\right)$ is independent from the particular potential) and produce the energy $Q\left(V-V^{\prime}\right)$. Next we take the machine used to create $Q$ back to the point with potential $V$, thus closing the cycle with a net energy gain $Q\left(V-V^{\prime}\right)$. The upshot is that in presence of gauge invariance, if the electric charge isn't preserved neither is energy.

Is it conceivable to describe the other interactions as well using gauge theories? In 1954 the Americans Chen Ning Yang and Robert Mills [9] tried to answer this question by constructing a theory of strong forces based on a non-Abelian gauge theory (Fig. 4).

The transformations we saw in electromagnetism are commuting, that is, they form an Abelian group: the result of applying two transformations one after the other doesn't change if we swap the order. Non-Abelian transformations don't commute. For strong interactions Yang and Mills suggested considering phase transformations more complicated than electromagnetic ones, given by non-commuting operators. They chose the group SU(2), seen earlier for the isospin, whose elements are transformations $\left(\psi_{p}\right.$, $\left.\psi_{n}\right) \rightarrow\left(\psi_{p}^{\prime}, \psi_{n}^{\prime}\right)=\mathrm{e}^{i g \tau \cdot \Lambda}\left(\psi_{p}, \psi_{n}\right)$, where $\Lambda$ is an arbitrary vector giving the axis of isospin rotation, $g$ is the coupling constant (playing a similar role to the electric charge in electromagnetism) and $\tau$ has as components the Pauli matrices $\tau_{1}, \tau_{2}, \tau_{3}$, which are non-commuting operators. The two components of the wavefunction $\left(\psi_{p}, \psi_{n}\right)$ describe protons and neutrons. In absence of electromagnetic interactions the global symmetry is such that an overall swap of protons and neutrons leaves the world unchanged. The fact that the transformation acts on two-dimensional wavefunctions implies we have to replace the phase angle of the electromagnetic gauge transformation with a $2 \times 2$ matrix, which renders the transformation non-Abelian. The transformation can be made local by requiring that $\boldsymbol{\Lambda}(\boldsymbol{r})$ varies with $\boldsymbol{r}$. The field generated by the local gauge transformation turned out to be mediated by massless bosons with both charged and neutral components, and having a coupling constant $g$ similar to the electric charge. Mediating bosons interacted with all particles having isospin, including bosons themselves: hence they acted both as carriers and as sources of the isospin field. This is an important distinction from Abelian gauge transformations: in non-Abelian situations field-carrying bosons retain the field's 'charge', in this case the isospin. There was one surprising feature, though: mediating bosons appeared to be massless, and since it was believed they had to have a mass (cf. the relation between the range of the interaction and the mass of mediating bosons in Sect. 3), this seemed to nullify the effort of Yang and Mills. Apparently.

Translated from the Italian by Simon George Chiossi
Acknowledgments I am grateful to Pietro Nastasi for the discussions from which the idea of this article originated. I thank Silvio Bergia for carefully and critically reading the first draft and for many useful suggestions.

\section{References}

1. Dirac, P. A. M.: The quantum theory of the emission and absorption of radiation. Proc. R Soc. Lond. 114(767), 243-265 (1927)

2. Fermi, E.: Quantum theory of radiation. Rev. Mod. Phys. 4, 87-132 (1932)

3. Fermi, E.: Tentativo di una teoria dell'emissione dei raggi 'beta'. La Ricerca Scientifica 4(2), 491-495 (1933)

4. Fermi, E.: Tentativo di una teoria dei raggi $\beta$. Nuovo Cimento 11, 1-19 (1934)

5. Fermi, E.: Versuch einer Theorie der $\beta$-Strahlen. Zeitschrift für Physik 88, 161-171 (1934)

6. Fermi, E., Yang, C.N.: Are mesons elementary particles? Phys. Rev. 76, 1739-1743 (1949)

7. Gell-Mann, M., Rosenbaum, R.P.: Elementary particles. Sci. Am. 197, 72-88 (1957)

8. Pais, A.: Inward Bound: of Matter and Forces in the Physical World. Clarendon Press, Oxford (1986)

9. Yang, C.N., Mills, R.L.: Conservation of isotopic Spin and isotopic gauge invariance. Phys. Rev. 96, 191-195 (1954)

10. Yukawa, H. On the interaction of elementary particles. Proc. Phys.Math. Soc. Jpn. 17, 48-57 (1935) [rpt. in Progress of Theoretical Physics Supplement n. 1, 1-10 (1955)]

\section{Author Biography}

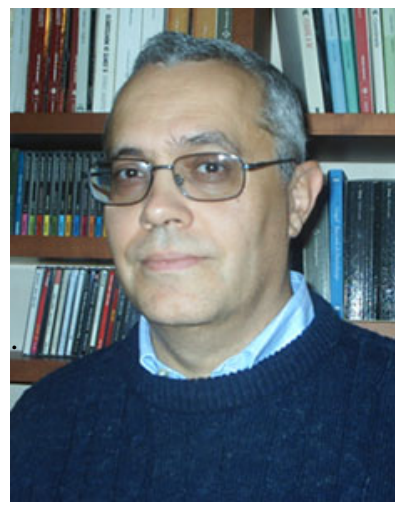

Giulio Maltese has been occupied for a number of years with the history of physics. His research has concerned the history of rational mechanics, relativity and twentieth-century physics in Italy, with a particular focus on Fermi and the group of physicists in Via Panisperna. 